encounters occurred. Those who had an open wound were more likely to receive pain medication vs those who did not have an open wound $(46 \%$ vs $7 \%, P<$ .001) There was a trend toward more pain medications administered to penetrating wounds vs blunt trauma $(28 \%$ vs $14 \%, P=.07)$. If an antibiotic was given, the patient was more likely to receive pain medication (66\% vs $19 \%$, $P<.001)$. Patients transported by AIREVAC were more likely to receive pain medication ( $35 \%$ vs $20 \%, P=.004$ ). If a tourniquet was placed, patients were more likely to receive pain medication $(47 \%$ vs $32 \%, P<.001)$. Medical officers were more likely to administer pain medications ( $43 \%$ vs $17 \%, P<.001)$.

Conclusions. - There were higher rates of pain medication administration for open wounds vs closed wounds, penetrating trauma vs blunt trauma, coadministration of antibiotics, AIREVAC vs ground evacuation, tourniquet placement, and treatment by a medical officer. A more detailed analysis by analgesia agent and whether the agents were within TCCC guidelines will be performed.

\section{Medical Problems of Casualties and Rescuers in the 2014 Volcanic Eruption in Japan}

Kazue Oshiro $^{1,2}$; Yuji Watanabe ${ }^{3}$; Tomikazu Muarakami ${ }^{2}$

${ }^{1}$ Cardiovascular Center Hokkaido-Ohno Hospital, Sapporo, Japan,

${ }^{2}$ The Hokkaido Prefectural Police, Sapporo, Japan, ${ }^{3}$ National Center for Mountaineering Education, Toyama, Japan

Introduction.- There are many active volcanoes worldwide that are popular destinations for tourists and trekkers. Mt. Ontake $(3067 \mathrm{~m})$, the second highest volcano in Japan and a popular mountain because it is a relatively easy climb, erupted without apparent warning and led to a heavy loss of life on September 26, 2014. Volcanic eruption disaster is an uncommon medical emergency and practical medical experience is limited in the medical literature.

Objective.-To consider the medical role and develop medical planning for emergencies during volcanic mountain disasters.

Methods. - Collected data included photos and movies provided by military, national police, and fire defense agency sources, in addition to publicly available data from the cabinet office.

Results.-Fifty-seven deaths, 6 losses, and at least 26 rescued. The primary cause of death was trauma by abrupt fallen rocks; victims died instantly. Survivors also experienced trauma due to abruptly fallen rocks. The location of dead bodies was concentrated on the mountain trails within a $1 \mathrm{~km}$-radius from the craters. More than 20,000 rescuers engaged in a search and rescue for 20 days, until winter set in. No deaths of rescuers occurred, but some had acute mountain sickness and hypothermia on missions.

Conclusions. - The volcano erupted violently and rapidly, leaving little or no time for people to escape or take shelter. Emergency medical care played a small role in this severe volcanic eruption. On the other hand, precautionary education about health hazards and medical care for rescuers is necessary and helpful.

\section{Canyoneering-Related Search and Rescue in Zion} National Park from 2005-2015

Heather Beasley ${ }^{1}$; Scott McIntosh ${ }^{2}$

${ }^{1}$ University of New Mexico School of Medicine, Albuquerque, NM,

USA, ${ }^{2}$ University of Utah Division of Emergency Medicine, Salt Lake City, UT, USA

Introduction and Background-Canyoneering is an increasingly popular outdoor activity within Zion National Park (ZNP) in Utah, yet the burden of this particular activity on search and rescue (SAR) resources is relatively unknown. ZNP maintains records from all SAR activations. Although a recent review of canyoneering rescue operations in Spain has been published, there has been no review of canyoneering SAR records in the United States until now.

Objectives.-To examine demographics of parties involved, relevant injury patterns, and cost of SAR response in canyoneering-related SAR activations in ZNP from 2005 to 2015 .

Methods.-ZNP provided 221 SAR records for incidents in which the activity was noted to be "canyoneering" between January 1, 2005 and December 31, 2015. Annual reports of all ZNP SAR activations from 2005 to 2015 also were obtained.

Results.-Out of 221 SAR activations from 2005 to 2015, there were 562 people involved. There was a male $(n=283)$ to female $(n=104)$ ratio of $2.7: 1$ for activations in which the sex of canyoneering parties was noted. One hundred eighteen people were recorded as ill or injured; there were 72 ill/injured men and 37 ill/injured women. The most common injuries were ankle injuries $(25.2 \%)$, followed by other lower extremity injuries (15.9\%), knee injuries $(11.2 \%)$, and illnesses attributable to environment/exposure (11.2\%). The most common age range of 378 canyoneers with recorded ages was $20-29(\mathrm{n}=137,36.2 \%)$. There were 11 total fatalities in the 10 -year period, with primary contributing factors to deaths being flash flooding $(n=7)$, falls $(n=3)$, and drowning vs exposure $(n=$ 1). Average cost of canyoneering-related SAR operations was $\$ 3483.33$ over the 10-year period.

Conclusions. - Canyoneering-related SAR contributes significantly to use of ZNP resources, with canyoneering-related SAR expenses making up $40.4 \%$ of the 10-year total SAR cost. Injury occurred in $51 \%$ of all canyoneering SAR activations in the 10-year period. Canyoneers should take measures to prevent lower extremity injuries as well as illness related to environment and exposure and should be prepared to manage such issues if they arise. Medical providers in national parks where canyoneering occurs should familiarize themselves with these injury patterns and methods to manage them in the backcountry.

Direct Measurement of Intracranial Pressure in Hypobaric Hypoxia: Implications for Acute Mountain Sickness

Justin Lawley $^{1,2}$; Louis Whitworth ${ }^{2}$; Benjamin Levine ${ }^{1,2}$

${ }^{1}$ Institute for Exercise and Environmental Medicine, Dallas, TX, USA, ${ }^{2}$ U.T. Southwestern Medical Center, Dallas, TX, USA

Introduction.-Despite decades of research, the pathophysiology of acute mountain sickness (AMS) is unknown. The classic hypothesis is that persistent and/or transient elevations in intracranial pressure (ICP) cause AMS.

Objective.-To make continuous, direct, invasive measurements of ICP alongside AMS (Lake Louise Questionnaire [LLQ]) in hypobaric hypoxia (4500 m).

Methods. - To date, we have recruited three men who had previously been cured of a hematologic malignancy and as such had an Ommaya reservoir implanted. A needle was placed inside the Ommaya reservoir for direct measurement of intraventricular pressure.

Results.-Participant 1: ICP at sea level was $13 \mathrm{~mm} \mathrm{Hg}$. After 9 hours at altitude, mean ICP remained normal $(14 \mathrm{~mm} \mathrm{Hg})$, but large fluctuations in ICP (>20 mm Hg) were observed, alongside symptoms of AMS (LLQ, 6). Administration of $100 \% \mathrm{O}_{2}$ stabilized ICP and symptoms resolved. With removal of $100 \% \mathrm{O}_{2}$, ICP pressure waves began and symptoms returned. Participant 2: ICP decreased slightly at altitude (SL, $11 \mathrm{vs}$ altitude, $6.6 \mathrm{~mm} \mathrm{Hg}$ ) and no pressure waves were observed over the first 9 hours. This subject reported only a very mild headache. During sleep, ICP fluctuated in opposition to oscillations in $\mathrm{SaO}_{2}$. The participant woke with mountain sickness (LLQ $=10$ ), which declined by 24 hours (LLQ $=4)$, where ICP was below the sea level value $(3 \mathrm{~mm} \mathrm{Hg}$ ). Participant 3: ICP at sea level was $16 \mathrm{~mm} \mathrm{Hg}$. After 9 hours at altitude, ICP was substantially elevated $(26 \mathrm{~mm} \mathrm{Hg})$ with symptoms of AMS (LLQ = 5). ICP was persistently $>20 \mathrm{~mm} \mathrm{Hg}$ while asleep, and the participant woke with muscle paralysis and severe AMS. Adminitration of $100 \% \mathrm{O}_{2}$ reduced ICP $(11 \mathrm{~mm} \mathrm{Hg})$ and resolved symptoms. This study was ended because of the possibility of mild cerebral edema.

Conclusions. - These preliminary observations suggest that persistent or transient elevations in ICP preceded the onset of AMS.

Funding: WMS Houston Grant (2013).

\section{Metal Accumulation in Horseshoe Crab (Limulus polyphemus) Eggs, Embryos, and Larvae From Potentially Contaminated Public Beaches}

Aaron Bakker ${ }^{1}$; Jessica Dutton ${ }^{2}$; Nicholas Santangelo ${ }^{1}$

${ }^{1}$ Hofstra University, Hempstead, NY, USA, ${ }^{2}$ Texas State University,

San Marcos, TX, USA

Introduction.- In many coastal ecosystems, humans and marine organisms are confronted with heavy metal contamination. Thus, such organisms could be used as a bioindicator to understand uptake pathways and the potential risks of these habitats to marine organisms and human health.

Objective.-To relate 13 different trace metal concentrations between surrounding habitat and accumulation levels across early life stages of horseshoe 
crabs, which use these beaches for development and which the public frequents on Long Island, NY.

Methods.-Metals were assessed in egg (prespawning), embryo (early postspawning), and larvae (late postspawning) stages. At all sites, eggs were extracted from adult females to obtain a baseline contamination level (ie, metals are transferred during egg formation). Embryos and larvae were collected from the sediment of each beach. Sediment and water were collected from all sites, and metal levels were compared to organism samples. Metal concentrations in all samples were determined using inductively coupled plasma mass spectrometry (ICP-MS). Descriptive statistics are reported and differences in life stages were analyzed using a Kruskal-Wallis test.

Results.-All trace metals accumulated, but to varying degrees based on life stage and beach. Essential metals that are required for physiological function showed significant accumulation from egg to larval stages for most sites $(P<$ .001). Nonessential metals, which can have toxic effects, showed significant accumulation from egg to larval stages for most sites $(P<.001)$.

Conclusions. - For a given metal, early stages related to sediment and water concentrations at some sites. Thus, local abiotic conditions may affect the uptake rate of each metal. We showed that metal bioaccumulation occurred at concentrations naturally found in habitats and suggested factors other than local levels of a contaminant should be considered when addressing the danger to organisms, including humans, that heavily use these areas.

Funding: WMS Hultgren Grant (2015).

\section{The Benefits of Wilderness Programs for Cancer Patients and Survivors: A Literature Review}

S. Terez Malka ${ }^{1}$; Tyler Warmack ${ }^{2}$

${ }^{1}$ Harvard School of Medicine, Boston, MA, USA, ${ }^{2}$ University of North Carolina School of Medicine, Chapel Hill, NC, USA

Introduction.-Many organizations promote wilderness experiences for cancer patients and survivors. Data suggest that moderate-to-vigorous exercise may reduce fatigue and depression in adults undergoing cancer treatment. The psychology literature suggests additional benefits of nature-based programming, including increased self-esteem and decreased anxiety. However, rigorous studies of the impact of wilderness-based programs are lacking.

Objective.-To evaluate the impact of wilderness programs on cancer patients and survivors.

Methods.-We searched PubMed, MEDLINE, CINAHL, and the Cochrane Central Register of Controlled Trials through March 2016. Search terms included adventure or wilderness or nature; therapy, rehabilitation, or treatment; and cancer. We reviewed the bibliographies of relevant articles for additional citations. Two authors independently analyzed the results for relevance and study design. Systematic reviews and controlled trials were selected for inclusion.

Results.-Forty-nine articles were identified: 38 were not relevant and 7 were case reports. Four studies were identified that met inclusion criteria, including 1 systematic review and 3 controlled trials. Outcomes of the controlled trials were heterogeneous, precluding meta-analysis. Two studies analyzed the impact of wilderness therapy programming on the physical activity levels of participants. The other 2 studies explored self-reported psychological benefits. Analysis of the 4 articles suggests benefits to program participants, including an increase in regular physical activity and improved perception of self-esteem, body image, quality of life, and mood. No studies assessed physical health outcomes or impact on disease progression.

Conclusions. - There are few rigorous studies evaluating the impact of adventure and wilderness programming on cancer patients and survivors, and no studies exploring medical effects. Preliminary data suggest positive, though potentially transient, benefits, including an increase in exercise frequency and improved self-esteem and mood. Given the popularity of these programs and potential benefits, further research is indicated to quantify the medical and psychological impact of wilderness programming on cancer patients and survivors.

Phase 3 Efficacy and Safety Results of Sufentanil Sublingual 30 mcg Tablet for Management of Acute Traumatic Pain in Emergency Medicine

James Miner ${ }^{1}$; Harold Minkowitz ${ }^{2}$; Zubaid Rafique ${ }^{3}$; Karen DiDonato ${ }^{4}$; Pamela Palmer ${ }^{4}$
${ }^{1}$ Hennepin County Medical Center, Minneapolis, MN, USA, ${ }^{2}$ Memorial Hermann Memorial City Medical Center, Houston, TX, USA, ${ }^{3}$ Ben Taub General Hospital, Houston, TX, USA, ${ }^{4}$ AcelRx Pharmaceuticals, Redwood City, CA, USA

Introduction.-Opioids often are considered standard-of-care treatment for acute pain associated with field trauma; however, limitations of these therapies have been well documented. Morphine is associated with erratic onset of analgesia when delivered intramuscularly (IM) and delayed side effects resulting from active metabolites, and fentanyl's rapid distribution demands frequent redosing. There remains a clinical need for rapid-acting, potent analgesics that do not require an invasive route of delivery. In collaboration with the Department of Defense, a sufentanil sublingual $30 \mathrm{mcg}$ tablet (SST) is in development for treatment of acute pain in battlefield and emergency trauma settings.

Objective.-To evaluate SST's safety and efficacy in managing moderateto-severe pain in the emergency department (ED).

Methods.-This is a multicenter, open-label, phase 3 study in up to 120 adult patients presenting to the ED with acute traumatic pain. SST was administered as needed but not more frequently than hourly. Efficacy was assessed by patient reports of pain intensity on an 11-point numerical rating scale $(0=$ no pain and $10=$ worst possible pain $)$. The primary efficacy variable was the summed pain intensity difference from baseline over the first hour (SPID1). Safety was assessed via vital signs and adverse event reporting (AEs).

Results.-Forty of the 120 patients have been enrolled to date and an interim analysis performed. Statistically significant and clinically meaningful reductions in pain intensity have been observed following a single dose of SST A low number of adverse events has been reported, with nausea and somnolence the most frequent (5\% each). All available safety and efficacy results will be included at the time of presentation.

Conclusions.-Sufentanil $30 \mathrm{mcg}$ tablets are in development for treatment of moderate-to-severe acute pain in an EM population. Early efficacy and tolerability results suggest that SST may offer a viable alternative to IM or IV dosing.

Funding: The late-stage development of this drug is being sponsored in part by the US Department of Defense.

\section{An Assessment of Diarrhea Among Long-Distance Backpackers in the Sierra Nevada}

Derek Meyer $^{1}$; Amber Costantino ${ }^{2}$; Susanne Spano ${ }^{1}$

${ }^{1}$ UCSF - Fresno, Fresno, CA, USA, ${ }^{2}$ California State University -

Fresno, Fresno, CA, USA

Introduction.- The John Muir Trail (JMT) is a 210-plus mile, longdistance backpacking trail in the Sierra Nevada mountain range of California Previous studies from the Appalachian Trail and Long Trail have found significant correlation between backpacker demographics and on-trail hiking behaviors and diarrhea incidence, ranging from $10.7 \%$ to $63 \%$. Incidence of diarrhea also has been shown to depend on age, sex, and regularity of standard backcountry hygiene practices. However, no studies to date have examined these variables among backpackers on the JMT.

Objectives.-To determine the significance of particular JMT backpacker demographics and hygiene compliance regularity with diarrhea incidence and severity.

Methods. - Survey data from long-distance backpackers who attempted a JMT trek in 2014 were assessed via statistical software for the significance of variables that might contribute to the incidence and severity of on-trail diarrhea These variables included age, sex, prehike body weight, prehike physical activity (hours/week), prehike self-assessed physical condition, previous hiking or backpacking experience (days in previous 10 years), solo or group backpacking itinerary, on-trail average pack-weight, on-trail hygiene compliance regularity (water filtration, hand hygiene, dish washing), and posthike difficulty assessment. Backpackers also reported their on-trail incidence and severity of diarrhea.

Results.-Of 737 valid responders, $16.4 \%$ reported experiencing diarrhea ( $82 \%$ with minimal or mild severity; $18 \%$ with significant severity). No statistical significance was found between diarrhea incidence as related to hiker demographics or compliance with standard hygiene recommendations. Regular hand sanitizer use was significantly correlated with having more severe diarrhea, but 